\title{
PERMISIONES Y ACTITUDES NORMATIVAS
}

\section{Introducción}

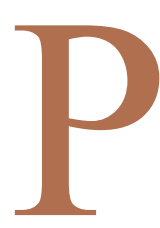

uede afirmarse que la existencia de los sistemas jurídicos depende de las actitudes del grupo social, i.e. aceptación, obediencia, etc.; ${ }^{1}$ y de las actitudes de las autoridades normativas ${ }^{2}$ del sistema. Una descripción apropiada de un sistema jurídico supone, entonces, dar cuenta de las relaciones existentes entre la configuración del sistema normativo y las actitudes de los sujetos y las autoridades normativas. En este trabajo analizaremos solamente la relación entre descripción de los sistemas jurídicos legislados y la descripción de las actitudes de las autoridades normativas (en adelante AN).

Sin pretensión de exactitud, podemos decir que las actitudes de las AN, en tanto que normativamente relevantes, se manifiestan en calificaciones deónticas de ciertas acciones o estados de cosas. Afirmar que la acción p es jurídicamente obligatoria presupone una AN que promulga una norma que obliga a $\mathrm{p}$, o bien, una norma que implica a $\mathrm{p} .^{3}$ Esto parece sugerir que la conformación del sistema se adecua a las actitudes de las AN. Probablemente es el expresivismo jurídico la corriente de filosofía del derecho más cercana a esta tesis.

En este trabajo, intentaremos mostrar algunas dificultades conceptuales involucradas en la relación sistema-actitud normativa. Para ello tomaremos como puntos centrales los conceptos de permisión ${ }^{4}$ y de sistema jurídico en tanto que sistema deductivo. ${ }^{5}$

${ }^{1}$ Hart, H., 1968, pág. 145. Bulygin, E., 1982 a, pág. 138.

${ }^{2}$ Von Wright, G., 1970, pág. 91. Bulygin, E., 1982 b, pág. 67. Alchourron, C., 1982, pág. 53. Vernengo, R., 1983, pág. 385 y 386.

3 Alchourron, C., y Bulygin, E., 1984 a, pág. 456. Alchourron, C., y Bulygin, E, 1976, pág. 9. Bulygin, E., 1985 a.

${ }^{4}$ Alchourron, C., y Bulygin, E., 1984 b. Alchourron, C., y Bulygin, E., 1985. Von Wright, G., 1970, pág. 100, Ross, A., 1968, pág. 116.

${ }^{5}$ Alchourron, C., y Bulygin, E., 1974, pág, 92. Alchourron, C., y Bulygin, E., 1979, pág. 67. 


\section{Distintos conceptos de permisión}

En tanto que descripciones del sistema normativo es necesario distinguir entre:

P1) Pw p = -p E Cn A. Esto significa que la conducta $p$ está permitida si y sólo si -p no pertenece al sistema $\mathrm{Cn} A$, y este sistema está compuesto sólo por normas de obligación.

P2) Ps $p=P p$ E Cn B. Esto significa que la conducta p está permitida si y sólo si existe una norma que permite la conducta $\mathrm{p}$ en el sistema $\mathrm{Cn} \mathrm{B}{ }^{6}$

En este punto encontramos las primeras dificultades. P1 es relativa a los actos no realizados por AN y $\mathrm{P} 2$ es relativa a la existencia de normas permisivas. Pero, P1 no correlaciona normas y actitudes sino la ausencia de normas y la ausencia de actitudes. Respecto a P2, es bien conocida en teoría del derecho la controversia sobre qué actitudes deben ser identificadas como permisiones. Entre los diversos análisis de este problema, nos detendremos en el que ofrecen Carlos Alchourron y Eugenio Bulygin (A-B, en adelante).

En su análisis, A-B expresan que no hay relaciones lógicas entre normas sino entre contenidos normativos o contenidos proposicionales. ${ }^{7} \mathrm{Al}$ exponer sobre la dinámica de los sistemas jurídicos, expresan que es necesario distinguir entre actos de promulgación y actos de rechazo. Rechazar es un tipo especial de acto ilocucionario, diferente de prescribir $u$ ordenar, el cual conduce a la eliminación de ciertos contenidos proposicionales del conjunto ordenado, de acuerdo con ciertas reglas. ${ }^{8}$

Cuando analizan el problema de las normas permisivas expresan: «el conjunto de las negaciones de los contenidos normativos permitidos... es formalmente idéntico al conjunto de proposiciones rechazadas ${ }^{9} \mathrm{y}$, consecuentemente, afirman que esto podría servir para justificar la pretensión expresivista de admitir sólo mandatos (normas de obligación), siempre que estén dispuestos a incorporar la noción de derogación. Con esta base, tenemos:

P3) Ps $p=$ AN! (-p) \& -p 17 Cn A. Esto significa que la AN ha permitido p si y sólo si ha rechazado $-\mathrm{p}, \mathrm{y}-\mathrm{p}$ no pertenece a $\mathrm{Cn} \mathrm{A}$.

${ }^{6}$ Alchourron, C., y Bulygin, E., 1984 b, pág. 353.

${ }^{7}$ Alchourron, C., y Bulygin, E., 1981, pág. 101.

${ }^{8}$ Alchourron, C., y Bulygin, E., 198 1, pág. 105. Alchourron, C., y Bulygin, E., 1984 a, pág. 460. Bulygin, E., 1985 b, pág. 185.

${ }^{9}$ Alchourron, C., y Bulygin, E., 1981, págs. 118 y 119. 
Con esta reformulación, al aceptar la actitud normativa de rechazar, tenemos una nueva manera de relacionar los sistemas normativos y los actos de las AN. P3 está vinculada a un conflicto actual o potencial de actitudes denominado «ambivalencia», el cual debe ser resuelto en favor del acto de rechazo para que P3 sea verdadera.

Denominaremos SSPR y SSPO, respectivamente, al conjunto de proposiciones del sistema normativo rechazadas y ordenadas por las AN. El conjunto de las consecuencias lógicas de los contenidos proposicionales rechazados (SSPR) limita la competencia de las AN de inferior jerarquía. Consecuentemente, es necesario identificar al SSPR para conocer la conformación del SSPO, ya que la promulgación de un contenido proposicional puede provocar un conflicto de ambivalencia según sea la conformación del SSPR. Por lo tanto, consideraremos que el sistema normativo está determinado por la identidad de los elementos de SSPR y SSPO.

P3 asegura que SSPR no es un conjunto vacío, y esto equivale a sostener que el sistema normativo es consistente (P3 implica a P1, la permisión fuerte o positiva implica a la permisión débil o negativa). Esto puede probarse de la siguiente manera:

a) Sea P3 verdadera.

b) $\mathrm{Si}$ el SSPO es inconsistente, entonces cualquier contenido normativo puede ser derivado en él.

e) -p puede ser derivado en un SSPO inconsistente, lo que contradice la hipótesis $a$.

A su vez, la inconsistencia del SSPR asegura que SSPO es un conjunto vacío. Pero es evidente que los juristas deben rechazar este intento de reconstrucción ya que:

- Distinguir entre permisión débil y fuerte es habitual en el análisis de sistemas inconsistentes e incompletos, y $\mathrm{P} 3$ borra parcialmente esta diferencia al implicar a P1. ${ }^{10}$

- Permitir el contenido proposicional $p$ y el contenido proposicional -p es equivalente a rechazar -p y a rechazar p. El SSPR es inconsistente y el SSPO es vacío. Sin embargo, los juristas no encuentran problemático permitir simultáneamente una conducta y su negación, denominando a tal situación «Facultamiento». ${ }^{11}$

Podría pensarse que el origen de estas dificultades es vincular

${ }^{10}$ Alchourron, C., y Bulygin, E., 1976, Cap. VII. Alchourron, C., y Bulygin, E., 1984 b, pág. 358 y 359 .

${ }^{11}$ Alchourron, C., y Bulygin, E., 1976, pág. 74. 
el acto de rechazo de un contenido normativo a la pertenencia de este contenido al sistema normativo; y que el concepto de permisión debe ser definido en términos de actos de rechazo solamente. De acuerdo con esto A-B expresan: «p está positivamente permitido por $\mathrm{x}$, si y sólo si -p (la negación u omisión de p) es ya sea explícita o implícitamente rechazada por $\mathrm{X} \gg .{ }^{12}$

P4) Ps $p=A N$ ! ex. $-p$ v imp -p. Esto significa que $p$ está positivamente permitida si hay una AN tal que rechaza explícita o implícitamente -p.

Para P4 lo único que cuenta es la actitud de AN, y es obvio que no implica a PI, consecuentemente P3 y P4 no son equivalentes. P3 puede tomarse como una descripción del contenido del sistema normativo y P4 como una descripción de actitudes de AN. ${ }^{13}$ Pero la descripción de las actitudes de las AN no garantiza la presencia o ausencia de los contenidos normativos objetos de promulgación o rechazo, y con ello, la relación entre actitud normativa y sistemas normativos pierde la nitidez que muestra al analizarse las normas de obligación.

\section{Permisión y jerarquía normativa}

Supongamos el siguiente sistema normativo $\mathrm{Cn} A$, que depende de las actitudes de $\mathrm{AN}$ y de $\mathrm{AN}-1$. Esta última es jerárquicamente inferior a $\mathrm{AN}$.

\section{(Ejemplo 1)}

\section{\{imagen}

En un momento T1, AN-1 rechaza q. A raíz de este acto, pertenecen al SSPR las consecuencias lógicas de los siguientes contenidos proposicionales:

a) Los contenidos proposicionales finalmente sustraídos del SSPO (Derogans).

${ }^{12}$ Alchourron, C., y Bulygin, E., 1984 a, pág, 461.

${ }^{13}$ No advertir con claridad esta diferencia lleva a Weinberger a sostener que rechazo y permisión son interdefinibles, afirmando que la diferencia entre ambas cosas radica solamente en la negación de los contenidos proposicionales. Cf. Weinberger, O., 1984, pág. 474. 
b) Aquellos contenidos proposicionales no pertenecientes a las $\mathrm{Cn}$ A que por sí solos o por su conjunción con las Cn A implican a los contenidos explícitamente rechazados.

c) Aquellos contenidos que no pertenecen a las $\mathrm{Cn}$ A pero implican a los contenidos explícitamente rechazados.

d) Aquellos contenidos, no pertenecientes a las $\mathrm{Cn}$ A, que implican a los contenidos identificados por el grupo $b$.

De acuerdo a P4, la negación de los contenidos que pertenecen a SSPR están permitidos. Sin embargo, es fácil ver que el sistema normativo no refleja las actitudes de las AN en, al menos, dos sentidos:

1. Existen ciertas actitudes normativas que son irrelevantes para la configuración del sistema. Al rechazar $\mathrm{q}$, implícitamente, $\mathrm{AN}-1$ ha rechazado los dos contenidos proposicionales que forman la base A del sistema $\mathrm{Cn} A$; pero solamente puede sustraer $\mathrm{p}$ de dicha base. La negación de $\mathrm{p}>\mathrm{q}$ está permitida P4 pero no es permitida P3 y, consecuentemente, los sujetos normativos no están autorizados por el sistema normativo a producir $\mathrm{p} \&-\mathrm{q}$.

2. Ciertas actitudes normativas son demasiado relevantes para la conformación del sistema. Supongamos que en un momento T2, AN-1 promulga q. Las únicas actitudes explícitas, luego de T0, fueron el rechazo y la promulgación de q. Sin embargo el contenido p continúa rechazado. Tal vez genere sorpresa advertir que el sistema normativo de T0 no es equivalente al de T2; aun cuando la única diferencia intuitiva entre ambos es el status de contenido derivado de q en T0 y el status de contenido formulado de q en T2. Sin embargo, la diferencia entre el sistema normativo de T0 y el de T2 no es simplemente la presentación de la base axiomática del SSPO, sino la diferente composición del conjunto de las consecuencias lógicas del SSPO y del SSPR. Generalizando, aun cuando sean idénticos los contenidos proposicionales que pertenecen al SSPO en T0 y T2, pueden no ser idénticos los contenidos que pertenecen al SSPR en esos mismos momentos. ${ }^{14}$

Podría sugerirse que así como el rechazo es la manera habitual de cancelar órdenes, promulgar es la manera habitual de cancelar rechazos. Pero todavía quedaría por responder si los distintos grupos de SSPR se incorporan al SSPO, o simplemente dejan de ser rechazados. Cualquier solución que elijamos nos alejará de la relación entre actitud normativa-sistema, a menos

${ }^{14}$ Bulygin, E., 1982 b, pág. 138. 
que respondamos que lo decisivo es lo que AN quiere hacer respecto de esos contenidos proposicionales. Lamentablemente, habrá demasiados casos en los que AN no conocía qué contenidos rechazaba implícitamente, y respecto de ellos no será posible señalar lo que quería $\mathrm{AN}$ al promulgar nuevamente ciertos contenidos.

\section{Inconsistencia y actitud normativa}

Es usual afirmar que $\mathrm{p}$ y $-\mathrm{p}$ son inconsistentes, ya que ambas no pueden ser verdaderas, pero de allí no podemos inferir que la pertenencia de p y -p al sistema normativo produzca inconsistencia normativa. Esto lo señalamos anteriormente (secc. II) al caracterizar al facultamiento. Para dar cuenta de ello, A-B señalan dos condiciones necesarias y suficientes para la inconsistencia normativa: ${ }^{15}$

1. Inconsistencia proposicional del SSPO.

2. Una dimensión pragmática: es el hecho de que estos contenidos proposicionales han sido ordenados lo que es decisivo para la caracterización de la inconsistencia normativa.

Este intento de caracterizar la inconsistencia normativa vuelve a vincular actitudes y pertenencia de proposiciones. Sin embargo, resta analizar qué sucede cuando la inconsistencia proposicional se da en el SSPR. Por lo que hemos visto, la pertenencia de una contradicción en el SSPR o en el SSPO producen similares efectos: la eliminación del subsistema correlativo. En un caso, todo contenido proposicional se encuentra ordenado y ninguno rechazado y en el otro caso, todo contenido proposicional se encuentra rechazado y ninguno ordenado. Independientemente de la plausibilidad de llamarle inconsistencia normativa a los casos de inconsistencia en el SSPR, un sistema en el que todo contenido esté rechazado, es tan inútil, a los fines de motivar conductas, como aquel en el que ningún contenido lo está. Cabe advertir, por último, otra importante consecuencia: El sistema normativo no depende de las actitudes de las AN. Si una de ellas ha tenido éxito al permitir p y -p, ha derogado, al mismo tiempo, todas las proposiciones del SSPO y, de allí en más, ningún contenido podría ser promulgado exitosamente por una $\mathrm{AN}$ jerárquicamente inferior. De no admitir esta consecuencia, resultaría que todo lo que está ordenado en el sistema normativo

\footnotetext{
${ }^{15}$ Alchourron, C., y Bulygin, E., 1984 a, pág. 458.
} 
está, a la vez, rechazado. El sistema normativo sería ambivalente, constituyendo este defecto un conflicto proposicional y no de actitudes. ${ }^{16}$

\section{Conclusiones}

- La relación entre actitudes normativas y sistemas jurídicos sólo puede expresarse sin dificultad en modelos simples: AN única (soberano) y normas de obligación (Modelo de Austin).

- La pluralidad de AN de diferentes jerarquías genera problemas al relacionarse con la caracterización del sistema jurídico como sistema deductivo.

- Admitir actos de rechazo implica admitir conjuntos rechazados; de esta manera, la identidad del sistema normativo depende de la identidad de los subsistemas SSPO y SSPR. El hecho de que el SSPO no se modifique luego de una acción normativa no preserva la identidad del sistema en caso de rechazo por adelantado y en la introducción de contenidos redundantes, ya que en ambos casos puede modificarse el SSPR. ${ }^{17}$

- El concepto de sistema jurídico momentáneo tiene que dar cuenta de las relaciones entre el SSPR y el SSPO.

- Los sistemas normativos que contienen proposiciones inconsistentes son defectuosos independientemente de la actitud de ordenar.

- Las distintas situaciones que reciben el nombre de «permisión» muestran que es preferible no reducir el concepto de permisión a un sólo tipo (P1). ${ }^{18}$ En otras palabras, es problemático reducir actos de permitir a estados de cosas permitidos.

- La caracterización de los sistemas jurídicos como sistemas deductivos puede servir como test de racionalidad de las $\mathrm{AN}$, pero no como base de una descripción de la relación real entre sistema y actitud.

${ }^{16}$ Esto parece sugerirse en Alchourron, C., y Bulygin, E., 1984 b, pág. 363, aun cuando esto entre en conflicto con lo que afirman en 1981, pág. 117.

${ }^{17}$ Bulygin, E., 1986, pág. 200. Stenius, E., 1982. Navarro, P., 1987, pág. 137.

${ }^{18}$ Guibourg, R., 1987, págs. 80 y 112. 


\section{BIBLIOGRAFÍA}

Alchourron, Carlos, «Normative order and derogation», en Deontic Logic, Computational Linguistic, and Legal Information Systems, A. Martino (ed.), vol. II, 1982, North-Holland Pc.

Alchourron, Carlos, y Bulygin, Eugenio, Introducción a la metodología de las ciencias jurídicas y sociales, 1974, Astrea.

-, «Sobre el concepto de orden jurídico», en Crítica, 8, 1976.

-, Sobre la existencia de las normas jurídicas, 1979, Venezuela.

-, «The expressive conception of norms», en New Studies in Deontic, R. Hilpinen(ed.), 1981, Reidel PC.

-, «Pragmatic foundations for a logic of norms», en Rechthstheorie, 15, 1984 a.

-, «Permissions and Permisive Norms», en Theorie der Normen. Festgabe für Ota

Weinberger, Krawietz, Schelsky, Winkler, Schramm (eds.), 1984 b.

-, «Libertad y autoridad normativa», en Boletín de la Asociación Argentina de Filosofía Jurídicay Social, año 3, núm. 26, 1985.

Bulygin, Eugenio, «Norms, normative propositions, and legal statements», en Contemporary philososphy. A new survey, vol. II, 1982 a, M. Nijhoff Publishers.

-, «Time and validity», en Deontic Logic. Computational Linguistics, and Legal Information Systems, A. Martino (ed.), vol. II, 1982b, North-Holand PC.

-, «Permissive Norms and Normative System», en Atti preliminari del II convegno internazionale di studi su logica, informatica, diritto, 1985 a, Firenze.

-, «Norms and Logic», en Law and philosophy, 4, 1985 b.

-, «Legal Dogmatics and the Systematization of Law», en Rechtstheorie. Beiheft, 10, 1986. Guibourg, Ricardo, El fenómeno normativo, 1987, Astrea.

Hart, Herbert, «El concepto del Derecho», 1968, Abeledo Perrot (trad. de G. Carrió).

Navarro, Pablo, «Redundancia: Notas para su análisis», en Análisis filosófico, 1987 (septiembre).

Ross, Alf, Directives and Norms, 1968, Routledge \& Kegan Paul.

Stenius, Erik, «Ross' pardox and well-formed codices», en Theoria, vol. XLVIII, 1982, part. 2.

Vernengo, Roberto, «Funciones normativas y voluntad de signo», en Anuario de Filosofía Jurídica y Social, vol. 3, 1983, Abeledo Perrot.

Weinberger, Ota, "On the meaning of norm sentences, normative inconsistency, and normative entailment», en Rechtsheorie, 15, 1984.

Von Wright, George, Norma y acción: una investigación lógica, 1970; Tecnos (trad, de Pedro García). 